\title{
Electrical Contact Formation in Micro FourPoint Probe Measurements
}

Folkersma, Steven; Bogdanowicz, Janusz; Petersen, Dirch Hjorth; Hansen, Ole; Henrichsen, Henrik Hartmann; Nielsen, Peter Former; Shiv, Lior; Vandervorst, Wilfried

Published in:

Physica Status Solidi. A: Applications and Materials Science (Online)

Link to article, DOI:

10.1002/pssa.201900579

Publication date:

2020

Document Version

Peer reviewed version

Link back to DTU Orbit

Citation (APA):

Folkersma, S., Bogdanowicz, J., Petersen, D. H., Hansen, O., Henrichsen, H. H., Nielsen, P. F., Shiv, L., \& Vandervorst, W. (2020). Electrical Contact Formation in Micro FourPoint Probe Measurements. Physica Status Solidi. A: Applications and Materials Science (Online), 217(5), [1900579].

https://doi.org/10.1002/pssa.201900579

\section{General rights}

Copyright and moral rights for the publications made accessible in the public portal are retained by the authors and/or other copyright owners and it is a condition of accessing publications that users recognise and abide by the legal requirements associated with these rights.

- Users may download and print one copy of any publication from the public portal for the purpose of private study or research.

- You may not further distribute the material or use it for any profit-making activity or commercial gain

- You may freely distribute the URL identifying the publication in the public portal 


\section{Electrical contact formation in micro four-point probe measurements}

Steven Folkersma*, Janusz, Bogdanowicz, Dirch H. Petersen, Ole Hansen, Henrik H. Henrichsen, Peter F. Nielsen, Lior Shiv and Wilfried Vandervorst

S. Folkersma, Dr. J. Bogdanowicz, Prof. Dr. W. Vandervorst

IMEC, Kapeldreef 75, B-3000 Leuven, Belgium.

E-mail: steven.folkersma@imec.be

Steven Folkersma, Prof. Dr. Wilfried Vandervorst

${ }^{\mathrm{b}}$ Instituut voor Kern en Stralingsfysika, KU Leuven, Celestijnenlaan 200D, B-3001 Leuven, Belgium.

Dr. D.H. Petersen

${ }^{\mathrm{c}}$ Department of Physics, Technical University of Denmark, Fysikvej, Bld. 307, DK-2800 Kgs. Lyngby, Denmark

Prof. Dr. O. Hansen

National Centre for Nano Fabrication and Characterization, DTU Nanolab, Technical University of Denmark, DK-2800 Kgs. Lyngby, Denmark

Dr. H.H. Hartmann, Dr. P.F. Nielsen, Dr. L. Shiv

CAPRES A/S, Scion-DTU, Building 373, DK-2800 Kgs. Lyngby, Denmark

Keywords: mirco four-point probe, finFET, electrical contact

Abstract - This paper describes the electrical contact formation between the electrodes of the micro four-point technique and a semiconducting sample. It is shown that the contact is formed in two stages: a voltage-induced electrical contact formation, followed by a currentinduced decrease in contact resistance. Moreover, a method is proposed allowing for precise control of the final contact resistance. Finally, it is demonstrated that the contacting process is similar on one-dimensional fin structures, where the demonstrated control of the electrical contact is needed when measuring on nanometer-wide fins in arrays with a pitch smaller than the electrode contact size.

\section{Introduction}

Ever since the transition from planar to three-dimensional transistor architectures such as the fin field-effect transistor (finFET) ${ }^{[1]}$ there has been a rising need for metrology solutions able This article has been accepted for publication and undergone full peer review but has not been through the copyediting, typesetting, pagination and proofreading process, which may lead to differences between this version and the Version of Record. Please cite this article as doi: 10.1002/pssa.201900579

This article is protected by copyright. All rights reserved 
to measure the electrical properties of nanometer-wide conducting features. ${ }^{[2]}$ Recently, it has been shown that the micro four-point probe $(\mu 4 \mathrm{pp})$ technique is able to measure the resistance in nanometer-wide fins. ${ }^{[3,4]}$ In order to perform such measurements with a high precision, all four electrodes are required to form low resistance contacts with the sample under investigation. ${ }^{[5]}$ When landing the electrodes on a semiconducting sample, however, the native oxides present on both the sample surface and the Ni-coated Si electrodes act as highly resistive barriers and prevent good electrical contact. ${ }^{[6]}$ Thus, every time the electrodes are landed on the sample surface, a lowly resistive electrical contact has to be created first. Generally, the electrical contacting is done by inducing an electrical bias to the sample with a current source, which results in the formation of an electrical contact under the electrodes. At present however, the understanding of this process is limited and the transients in induced voltage and injected current make the electrical contact formation hard to control.

In this paper, we show experimentally that the electrical contact between the $\mu 4 \mathrm{pp}$ electrodes and a blanket $\mathrm{Si}$ sample is formed in two separate phases; (1) a voltage-induced electrical contact formation and (2) a current-induced contact improvement (i.e. a further reduction of the contact resistance). Moreover, for phase 2, we propose a new method allowing for excellent control of the contact resistance of the created electrical contacts. Finally, we show that the electrical contact formation is similar on more confined structures such as fins, where the precise control of the electrical contact is needed for measuring single fins in dense arrays. $^{[4]}$

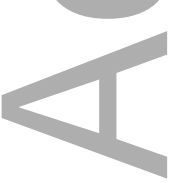

\section{The electrical contacting process}

As mentioned in the introduction, the formation of an electrical contact can be separated into two distinct phases. First, we show that a voltage-induced dielectric breakdown of the native oxide between the $\mu 4 \mathrm{pp}$ electrodes and the sample forms the initial electrical contact. Then, 
we demonstrate that a current can be used to further lower the contact resistances, such that an ohmic contact is created. Here, we start from the more general case of a Si blanket layer with a B implant $\left(3 \times 10^{15} \mathrm{~cm}^{-2}, 5 \mathrm{keV}\right.$ with a $1150{ }^{\circ} \mathrm{C}$ laser anneal $)$ and a deep lowly $\mathrm{n}$-doped well as a sample. The description in this paper as well as Figure 1a is only focused on two of the four electrodes needed for a micro four-point probe measurement. Needless to say, the extra two electrodes will have to undergo a similar contact formation process before an actual four-point measurement can be performed.

\subsection{Voltage induced contact formation}

In the first phase of the contacting process, the system attempts to inject a sinusoidal current $I_{\text {in }}$ through electrodes 1 and 2 landed on the Si sample surface, which has a native oxide layer (i.e. $\mathrm{SiO}_{2}$ ) of $1-2 \mathrm{~nm}$ (as illustrated in Figure 1a), grown by exposing the $\mathrm{Si}$ surface to air $(t>$ month). ${ }^{[6]}$ In this paper, we use $I_{\text {in }}=10 \mu \mathrm{A}$ with a $7 \mathrm{~Hz}$ frequency. When injecting the current $I_{\text {in }}$ a high load resistance (i.e. $R_{\text {load }} \gg R_{\text {sample }}$ in Figure 1a) is present between the sample and the current source. While $I_{\text {in }}$ is being applied, we can measure both the output voltage over the electrodes, i.e. $V_{\text {out }}=I_{\text {in }} * R_{\text {series }}$ (with $R_{\text {series }}=R_{1}+R_{\text {sample }}+R_{2}$ ) and the contact resistances $R_{1}$ and $R_{2}$, i.e. the resistances under electrodes 1 and 2 respectively (Figure 1b)

Note that the measurement of $R_{1}$ and $R_{2}$ is done using the extra two electrodes 3 and 4 (not shown in Figure 1a) of our micro-probes after forming the corrrespoding electrical contacts $R_{3}$ and $R_{4}$ with a high current ( $>500 \mu \mathrm{A}$, see Figure $\mathbf{2 b}$ in the next section) to ensure a low contact resistance (i.e. $R_{3}, R_{4} \ll<R_{1}, R_{2}$ ). Here, since the Si sample is highly doped, $R_{\text {sample }}$ is much lower than the contact resistances (i.e. $\left.R_{\text {sample }}<<R_{1}, R_{2}, R_{3}, R_{4}\right)^{[3][4]}$ and can thus be neglected, making that all contact resistances can be ontained using a series of independent two-point measurements. For example, $R_{1}$ is extracted using $R_{1}=0.5 *\left(R_{1-3}+R_{1-4}-R_{3-4}\right)$, 
where $R_{1-3}$ is the two-point measurements between electrode 1 and 3 etc. $R_{2}$ is extracted in a similar fashion. Furthermore, to make sure $R_{1}$ and $R_{2}$ can be measured during the short time frame in Figure 1b, the applied current signal is stopped at a specific time. For instance, to measure $R_{1}$ and $R_{2}$ at $t=2.5 \mathrm{~ms}$, the applied current is stopped at $t=2.5 \mathrm{~ms}$, after which $R_{1}$ and $R_{2}$ are measured as described above. This process is then repeated for each of the data points in Figure 1b. The contact resistances reported in this paper represent the median values of a set of 50 measurements done in subsequent electrode landings, since several large outliers are generally present. The corresponding error bars represenent the $25^{\text {th }}$ and $75^{\text {th }}$ percentile.

As can be seen in Figure 1b, $V_{\text {out }}$ goes through a series of two spikes when trying to apply $I_{\text {in. }}$. These two voltage spikes correspond quite precisely to the moment that respectively $R_{l}$ (blue triangles) and $R_{2}$ (red circles) drop from $\sim 10 \mathrm{M} \Omega$ to $\sim 10-100 \mathrm{k} \Omega$. During the first spike, i.e. the first $2.5 \mathrm{~ms}$ in Figure $\mathbf{1 b}, \mathrm{V}_{\text {out }}$ increases as the tool tries to apply $I_{\text {in. }}$ In this time frame both $R_{1}$ and $R_{2}$ are still very high, indicating that the oxide under both electrodes 1 and 2 is intact. However, after $V_{\text {out }}$ reaches $\sim 4.5 \mathrm{~V}$ (i.e. at time $\sim 2.5 \mathrm{~ms}$ ), there is a sudden drop in $R_{l}$ to $\sim 10^{5} \Omega$, while $R_{2}$ remains very high. As a consequence, there is a corresponding rapid drop in $V_{\text {out }}$ We understand this first peak as indicative of the dielectric breakdown of the oxide under electrode 1 , creating a conductive path between the electrode and the sample, while the oxide under electrode 2 is still intact. ${ }^{[6,7,8]}$ Quantitatively, since now $R_{1} \ll R_{2}$, the total series resistance drops by a factor $\sim 2$, thus resulting in $V_{\text {out }}$ dropping by a factor $\sim 2$ as well.

Next, after contact 1 is created (i.e. at time $>2.5 \mathrm{~ms}$ ), $\mathrm{V}_{\text {out }}$ increases again as the system tries to supply $I_{\text {in }}$ while $R_{2}$ is still very high. Then, when the voltage reaches $\sim 4.5 \mathrm{~V}$ (i.e. at time $\sim 4.5 \mathrm{~ms}$ ), $R_{2}$ suddenly drops to $\sim 10^{5} \Omega$ at its turn, expectedly corresponding to a drop in $V_{\text {out }}$. This indicates the dielectric breakdown of the oxide under electrode 2 . The exact breakdown voltage required will depend on the local oxide thickness ${ }^{[9]}$ and electrode geometry and might 
vary for the different electrodes, as can be seen by the slightly different breakdown voltages in Figure 1b. After the second peak, the electrical contacts under electrodes 1 and 2 are formed.

It is important to mention that the exact mechanism leading to the creation of these electrical contacts is not fully understood as yet. However, a possible explanation is the formation of conductive filaments in the oxide between the electrodes and the sample. Indeed, filamentbased switching behaviour has been observed in similar material stacks, i.e. $\mathrm{Ni}-\mathrm{SiO}_{2}-\mathrm{Si}$. ${ }^{[10,11,12]}$ Such a process could also explain why $R_{l}$ breaks down first, as the filament formation is favoured when a positive field is applied from the metal towards the semiconductor. The electric field is indeed not symmetric for both electrodes as it points into the sample under electrode 1 and out of the sample under electrode 2 (red arrows in Figure 1a). ${ }^{[13]}$ Further support for the filament formation process in our case is the fact that the resistance of such filaments also depends strongly on the current during their formation. ${ }^{[14,15]}$ As we show in the next section, the contact resistances $R_{1}$ and $R_{2}$ can be reduced by $\sim 1-2$ orders of magnitude by applying a current into the newly created electrical contacts.

\subsection{Current induced contact improvement}

As mentioned above, we now demonstrate that by applying a current through the electrical contacts after the voltage-induced contact formation, $R_{1}$ and $R_{2}$ can be further reduced. One of the main reasons why we need a low contact resistance is that an otherwise high contact resistance is observed as a non-linear contact, which in turn leads to inaccurate measurements. Typically, after both contacts have been formed (i.e. at $4.5 \mathrm{~ms}$ in Figure 1b), the load for the current source (i.e $R_{\text {load }}+R_{1}+R_{\text {sample }}+R_{2}$ ) drops from $\sim 10 \mathrm{M} \Omega$, as both $R_{1}$ and $R_{2}$ become much smaller $(\sim 10-100 \mathrm{k} \Omega)$. As the system cannot respond fast enough, this leads to a short $(\sim \mu \mathrm{s})$ current spike running through the electrodes, until $V_{\text {out }}$ is adapted to the 
new output resistance. Unfortunately, the magnitude of the current spike cannot be measured directly and moreover depends on $R_{1}$ and $R_{2}$ during the voltage transient (i.e. at $t=4.5 \mathrm{~ms}$ in Figure 1b), making the current spike hard to predict. However the result of this current transient can be seen and is that the contact resistances decrease further (in line with the behaviour seen for filamentary devices ${ }^{[13,14]}$ ), as is observed by the further reduction of $R_{l}$ after the second voltage peak (i.e. at $4.5 \mathrm{~ms}$ in Figure 1b).

To demonstrate this in more detail and to highlight the role of the current on the contact resistance more clearly, we measure $R_{1}$ and $R_{2}$ as a function of an extra applied current after the initial voltage-induced contact formation of $R_{l}$ and $R_{2}$ (see Figure 2a). Here, we can vary the magnitude of the current spike during the voltage-induced contact formation by varying the load resistance on the electrodes, i.e. $R_{\text {load. }}$ Indeed, it is expected that a low current spike is generated when a high load resistance $R_{\text {load }} \gg R_{\text {sample }}$ is used (as was done for the data in Figure 1b) whereas a much larger current spike is expected when using a low load resistance

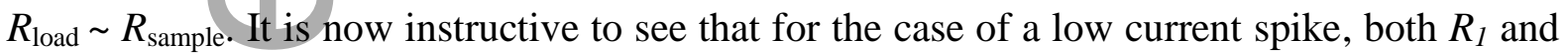
$R_{2}$ (i.e. red circles and blue triangles in Figure $\left.2 \mathbf{2 a}\right)$ are initially much higher $(\sim 100 \mathrm{k} \Omega)$ than for the case of a high current spike $\left(\sim 1 \mathrm{k} \Omega\right.$, dashed lines in Figure 2a). Moreover, both $R_{1}$ and $R_{2}$ can be lowered ( 1-2 orders of magnitude) to the value obtained with a high current spike by applying an additional current. Vice versa, when applying similar currents for the case of a high current spike, no further change in resistance is observed.

It is also interesting to evaluate the quality of the contacts after these procedures. Indeed, in a four-point measurement, where a sinusoidal current is injected between two electrodes and the voltage drop is measured by the other two electrodes, a non-zero phase shift $\left(\sim 0^{\circ}\right)$ between these two signals is indicative of a high resistive, non-linear contact under one or more of the electrodes, which can be observed when measuring $V_{\text {out }}$ as a function of time (not shown). ${ }^{[5]}$ To evaluate whether the formed contacts are indeed resistive, the phase shift can 
be measured while applying the current ramp through the electrodes. Here, the phase shift is measured in the regular four-point setup using all four electrodes, requiring the contacting process used for electrodes 1 and 2 to be repeated on electrodes 3 and 4 . As expected for the case of a low current spike (i.e. $R_{\text {load }} \gg R_{\text {sample }}$ ), the phase shift decreases with increasing current (see circles Figure $2 \mathbf{b}$ ), corresponding to the decreasing contact resistances $R_{l}$ and $R_{2}$ (and thus $R_{3}$ and $R_{4}$ ) in Figure 2a. Moreover, the phase shift approaches zero (i.e. the contacts are resistive) after applying a high enough current (i.e. $\sim 400 \mu$ A). Subsequently, as expected for the case of a high current spike (i.e $R_{\text {load }} \sim R_{\text {sample }}$ ), the phase shift remains $\sim 0^{\circ}$ since $R_{1}$ and $R_{2}$ (and thus $R_{3}$ and $R_{4}$ ) are already low (see triangles Figure $2 \mathbf{b}$ ).

These results clearly indicate that the final contact resistance is controlled by the maximum current either during or after the voltage-induced contact formation process and that ohmic contact can be achieved. As the current spike is nevertheless hard to predict or control, our observations suggest a simple approach for a finer control of the final contact resistances. Indeed, in the case of using a high load resistance $\left(R_{\text {load }} \gg R_{\text {sample }}\right)$ to suppress the current spike during the voltage-induced contact formation and a subsequent injection of a current with a desired magnitude, the contact resistances can be reduced in a controlled fashion.

\section{Electrical contact on fin structures}

Finally, the same electrical contacting process can also be used on confined structures such as fins. Figure 3a shows the contact resistance under electrodes $1\left(R_{1}\right.$, red circles) and $2\left(R_{2}\right.$, blue triangles) when measuring on an $80 \mathrm{~nm}$ wide Si fin with a B implant $\left(3 \times 10^{15} \mathrm{~cm}^{-2}, 5\right.$ $\mathrm{keV})$ and a $1150{ }^{\circ} \mathrm{C}$ laser anneal. Here, we used a high load resistance $\left(R_{\text {load }} \gg R_{\text {sample }}\right)$ during the voltage-induced contact formation. Similar to the blanket Si case discussed before, $R_{1}$ and $R_{2}$ decrease as a function of current from initially $\sim 2 \times 10^{5} \Omega$ to $\sim 2-5 \mathrm{k} \Omega$. Correspondingly, the phase shift during regular four-point measurement is also observed to decrease with increasing current (Figure 3b). Note that here the current needed to create an 
ohmic contact (i.e. phase shift $\sim 0^{\circ}$ at $\sim 40 \mu \mathrm{A}$ ) is much lower compared to the current needed when measuring on a blanket layer (i.e. $\sim 400 \mu \mathrm{A}$ ). At present the physical mechanism for this difference is not yet understood and needs further exploration as a function of fin width. Interestingly, with our proposed method of using a controlled current to lower the contact resistances, an excellent control of the electrical contact formation and the current during this process is achieved, which is required when trying to contact single fins in fin arrays with a pitch smaller than the electrode contact size. Indeed, it was shown that the maximum current -

during the electrical contact formation has to be carefully controlled in order to make sure a the electrical contacting remains isolated to a single fin. ${ }^{[4]}$

\section{Conclusions}

In this paper, we discussed the electrical contact formation between the $\mu 4 \mathrm{pp}$ electrodes and the sample. First, we showed an initial electrical contact is created by inducing the dielectric breakdown of the native oxide of the sample under the $\mu 4 \mathrm{pp}$ electrodes. Secondly, we demonstrated that the contact resistance under the electrodes can be further improved (i.e. decreased) by running a current through the electrodes. Moreover, we proposed a method which allows for excellent control of the contact resistance, which is essential when trying to contact on single fins in dense arrays. Additionally, this method could also be used to investigate the electrical contacting procedure on other materials such as $\mathrm{Ge}$, where the difference in native oxide properties might change the electrical contacting process. ${ }^{[16]}$

\section{Acknowledgements}

This project has received funding from the European Union's Horizon 2020 research and innovation program under grant agreements nrs. 688225 and 692527.

\section{References}

[1] D. Hisamoto, Wen-Chin Lee, J. Kedzierski, H. Takeuchi, K. Asano, C. Kuo, E. Anderson, Tsu-Jae King, J. Bokor, Chenming Hu, IEEE Trans. Electron Devices, 2000, 47, 2320-2325. 
[2] W. Vandervorst, C. Fleischmann, J. Bogdanowicz, A. Franquet, U. Celano, K. Paredis, A. Budrevich, Mater. Sci. Semicond. Process., 2017, 62, 31-48.

[3] J. Bogdanowicz, S. Folkersma, S. Sergeant, A. Schulze, A. Moussa, D.H. Petersen, O. Hansen, H.H. Henrichsen, P.F. Nielsen, W. Vandervorst, Phys. Stat. Sol. (a), 2018, 215, 1700857.

[4] S. Folkersma, J. Bogdanowicz, A. Schulze, P. Favia, D.H. Petersen, O. Hansen, H.H. Henrichsen, P.F. Nielsen, L. Shiv, W. Vandervorst, Beil. Jour. of Nanotech., 2018, 9, 1863-1867.

[5] D. H. Petersen, Micro Four-Point Probe and Micro Hall Effect, Ph.D.Thesis, Danish Technical University, 2009.

[6] M. Morita, T. Ohmi, E. Hasegawa, A. Teramoto, J. Appl. Phys., 1990, 29, L2392.

[7] J.F. Verweij, J.H. Klootwijk, Microelectron. J., 1996, 27, 611-622.

[8] S. Lombardo, J.H. Stathis, B.P. Linder, K.L. Pey, F. Palumbo, C.H. Tung, J. Appl. Phys., 2005, 98, 121301.

[9] N. Klein, H. Gafni, IEEE Trans. Electron Devices, 1966, 13, 281-289.

[10] K.-L. Lin, Y.-M. Tseng, J.-H. Lin, J. Shieh, Y.-J. Lee, T.-H. Hou, T.-F. Lei, 4th IEEE International Nano Electronics Conference, IEEE, Tao-Yuan, Taiwan, 2011: pp. 1-2.

[11] K.-L. Lin, T.-H. Hou, Y.-J. Lee, J.-W. Chang, J.-H. Lin, J. Shieh, C.-T. Chou, T.-F. Lei, W.-H. Chang, W.-Y. Jang, C.-H. Lin, Jpn. J. Appl. Phys., 2013, 52, 031801.

[12] M.B. Gonzalez, J.M. Rafí, O. Beldarrain, M. Zabala, F. Campabadal, IEEE Trans. Device Mater. Reliab., 2014, 14, 769-771.

[13] H. Jiang, X.Y. Li, R. Chen, X.L. Shao, J.H. Yoon, X. Hu, C.S. Hwang, J. Zhao, Sci. Rep., 2016, 6, 22216.

[14] R. Waser, M. Aono, Nat. Mater., 2007, 6, 833-840.

[15] U. Russo, D. Kamalanathan, D. Ielmini, A.L. Lacaita, M.N. Kozicki, IEEE Trans. Electron Devices 2009, 56, 1040-1047.

[16] S.K. Sahari, H.Murakami, T. Fujioka, T. Bando, A. Ohta, K. Makihara, S. Higashi, S. Miyazaki, Jpn. J. Appl. Phys., 2011, 50, 04DA12. 
(a)

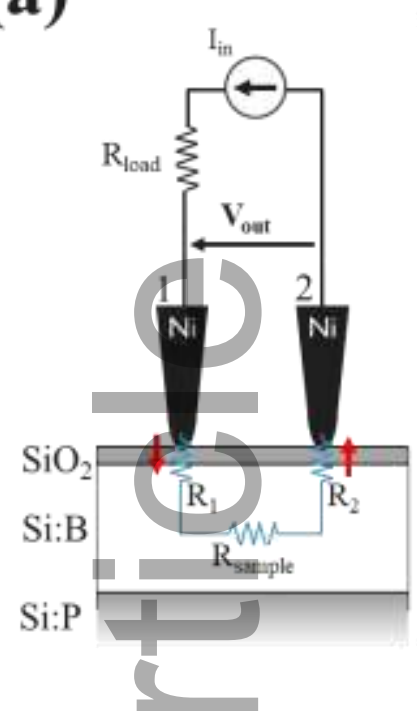

(b)

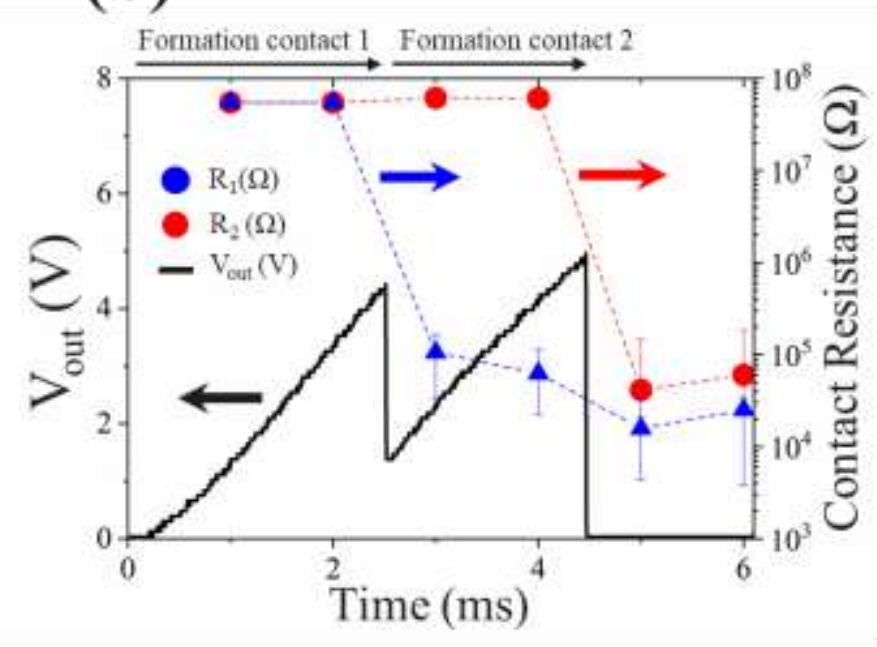

Figure 1: (a) Schematic of two of the four $\mu 4 \mathrm{pp}$ electrodes, consisting of Ni coated Si cantilevers, landed on the surface of a Si sample, with a native oxide layer of 1-2 nm. A current $\mathrm{I}_{\mathrm{in}}$ is applied between the two electrodes in order to create an electrical contact with the sample. $R_{1}$ and $R_{2}$ represent the contact resistances between the sample and electrodes 1 and 2, respectively, while $R_{\text {sample }}$ is the sample resistance and $R_{\text {load }}$ is an external series resistance. The red arrows show the direction of the electric field at the contact as the system tries to inject a current from electrode 1 to electrode 2. (b): Output voltage of the current source during the electrical contact formation process, as the tool tries to apply $I_{\text {in. }}$ The contact resistances for each of the electrodes, $R_{1}$ and $R_{2}$, during the contact formation process are also shown. The two spikes in $\mathrm{V}_{\text {out }}$ while $\mathrm{I}_{\text {in }}$ is being applied, correspond to the moment when contact resistances $\mathrm{R}_{1}$ and $\mathrm{R}_{2}$ drop from $\sim 10 \mathrm{M} \Omega$ to $\sim 10-100 \mathrm{k} \Omega$, respectively. Arrows indicate the corresponding axes of the data points.

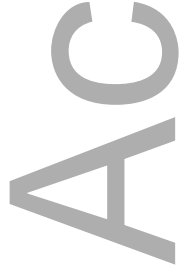



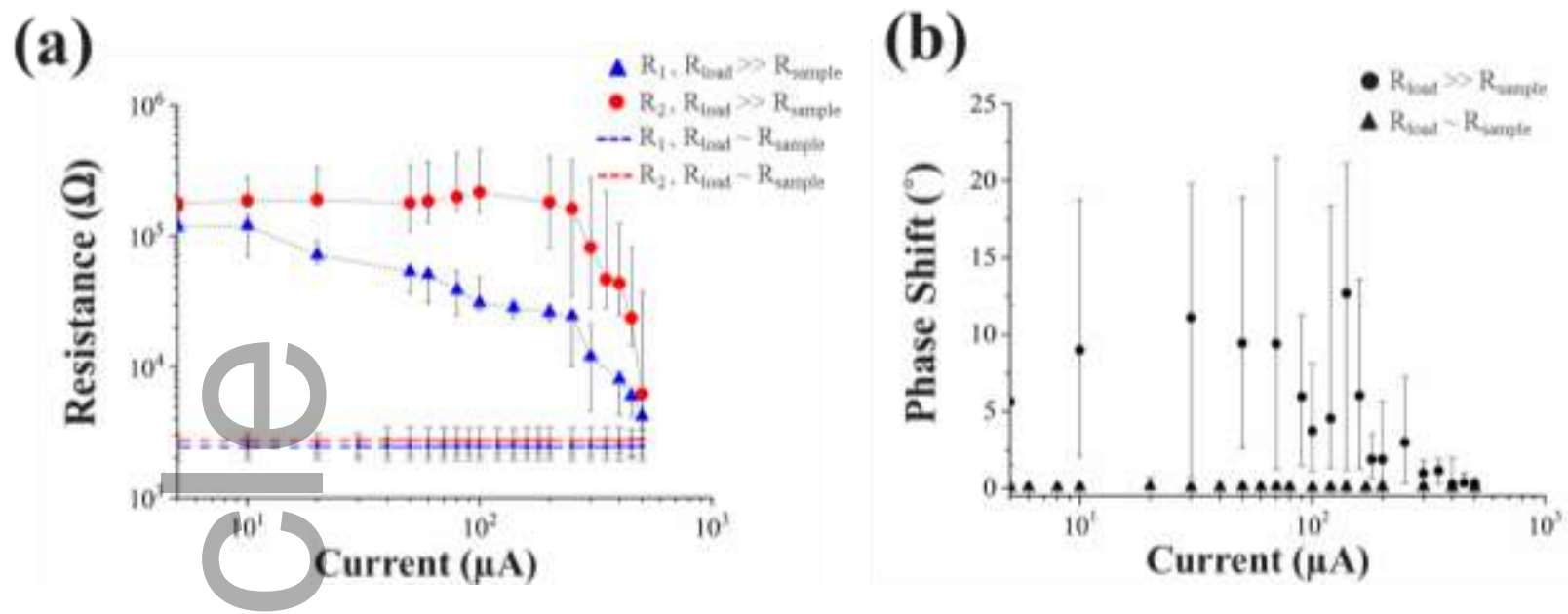

Figure 2: (a) Contact resistances under electrodes 1 and 2, i.e. $R_{1}$ and $R_{2}$, as a function of applied current after the voltage-induced contact formation. The data is shown for both the case of using $\mathrm{R}_{\text {load }}$ > $\mathrm{R}_{\text {sample }}$ (red circles and blue triangles) or $\mathrm{R}_{\text {load }} \sim \mathrm{R}_{\text {sample }}$ (red and blue dashed lines) during the voltage-induced contact forming. (b) Phase shift between injected current and measured voltage signal using the electrodes in regular four-point setup using all four electrodes (after also forming electrical contacts under the extra two electrodes), both for the case of $R_{\text {load }}>R_{\text {sample }}$ and $R_{\text {load }} \sim$ $\mathrm{R}_{\text {sample. }}$. The phase shift is $\sim 0^{\circ}$ (i.e. the system is resistive) only when $\mathrm{R} 1$ and $\mathrm{R} 2$ are low $(\sim \mathrm{k} \Omega$ ).
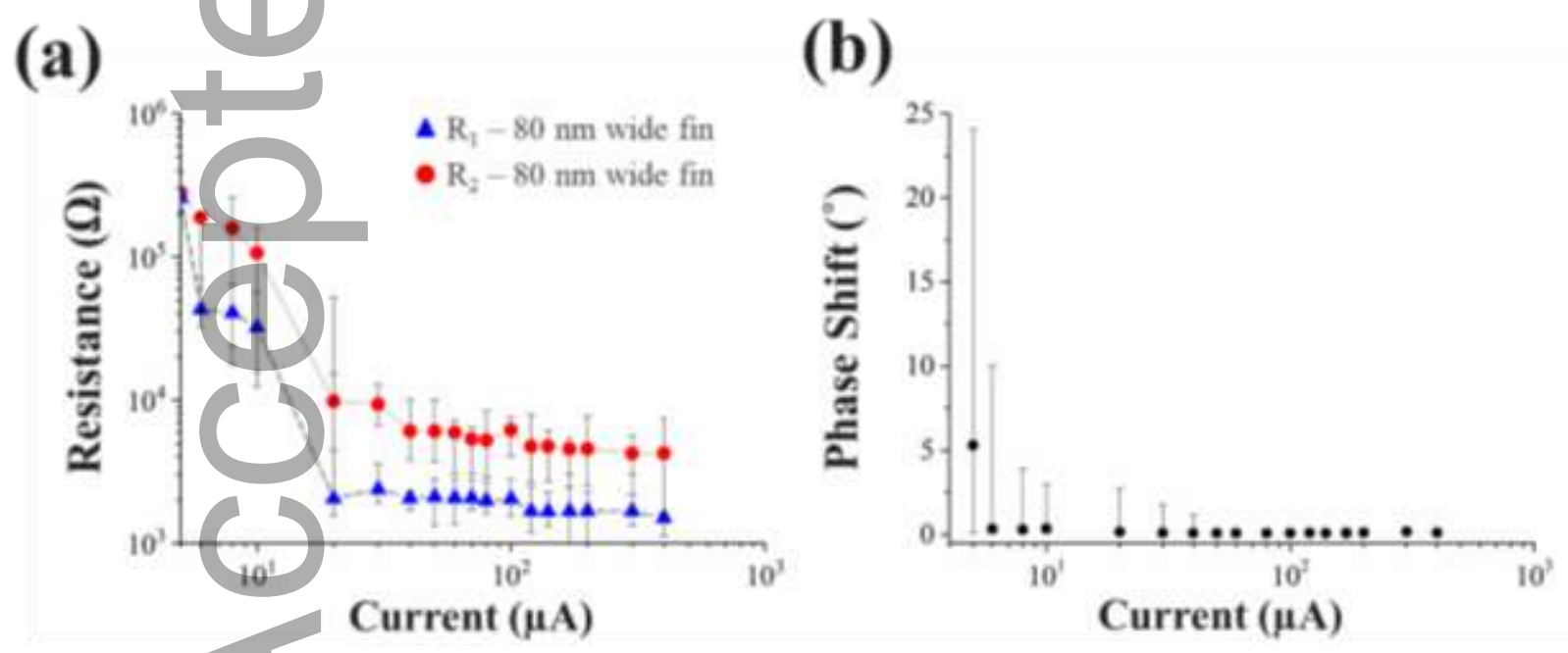

Figure 3: (a) Contact resistances under electrodes 1 and 2, i.e. $R_{1}$ and $R_{2}$, as a function of applied current when using $80 \mathrm{~nm}$ wide Si fins with a B $\left(3 \times 10^{15} \mathrm{~cm}^{-2}\right.$ with a $1150{ }^{\circ} \mathrm{C}$ laser anneal $)$ implant as a sample. Similar to a $\mathrm{Si}$ blanket layer, $\mathrm{R}_{1}$ and $\mathrm{R}_{2}$ decrease as a higher current is applied through the contacts. (b) Phase shift between the injected current and measured voltage signal using the electrodes in regular four-point setup. 


\section{Table of contents}

This paper presents a detailed study on the electrical contact formation between the electrodes of the micro four-point technique and a semiconducting sample. Additionally, we propose an electrical contact formation method which allows for precise control of the final contact resistance, which is essential when trying to measure on nanometer-wide fins dense arrays.

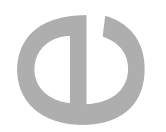

Keyword: micro four-point probe

Steven Folkersma*, Janusz, Bogdanowicz, Dirch H. Petersen, Ole Hansen, Henrik H. Henrichsen, Peter F. Nielsen, Lior Shiv and Wilfried Vandervorst

Electrical contact formation in micro four-point probe measurements

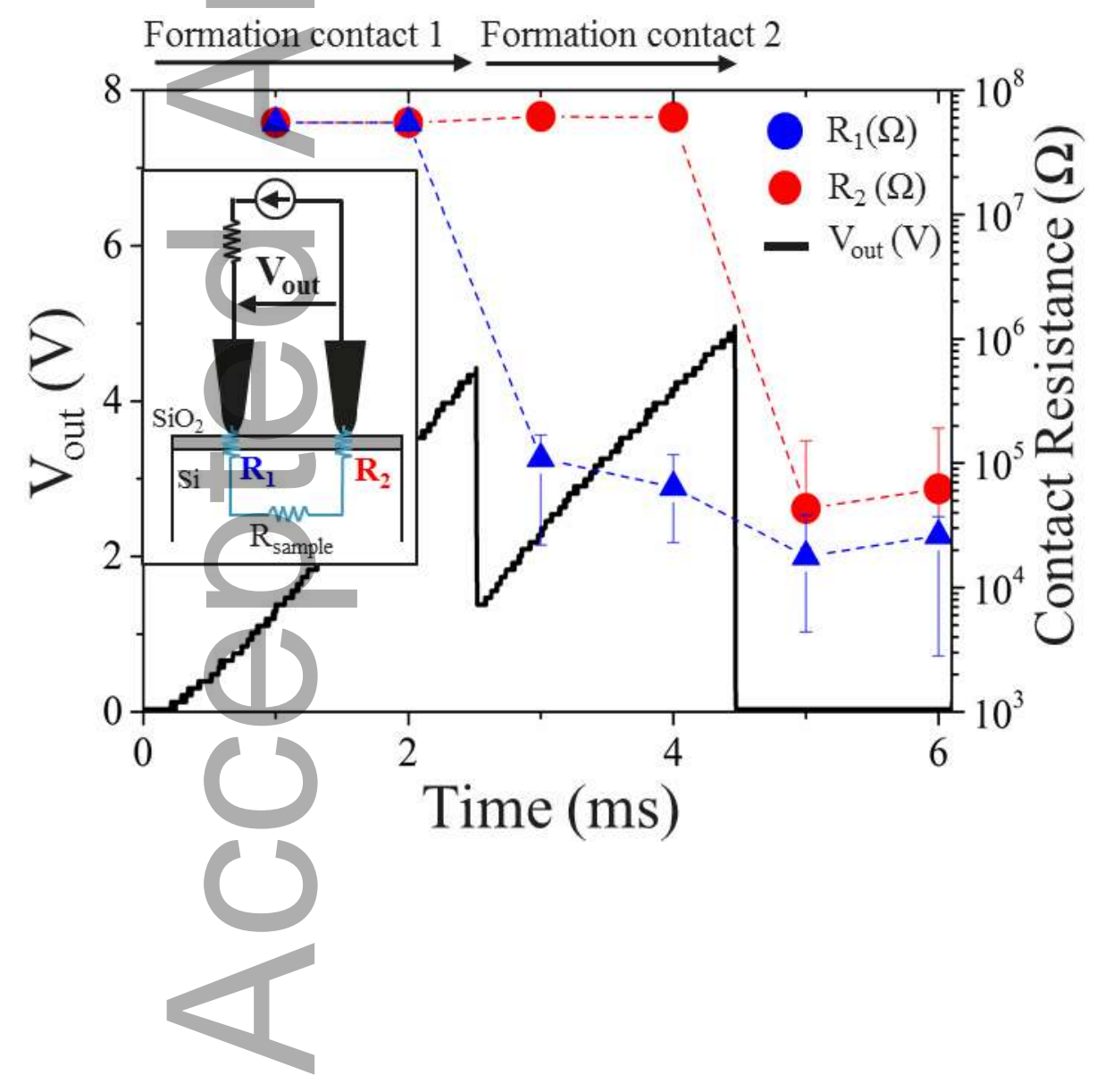

